
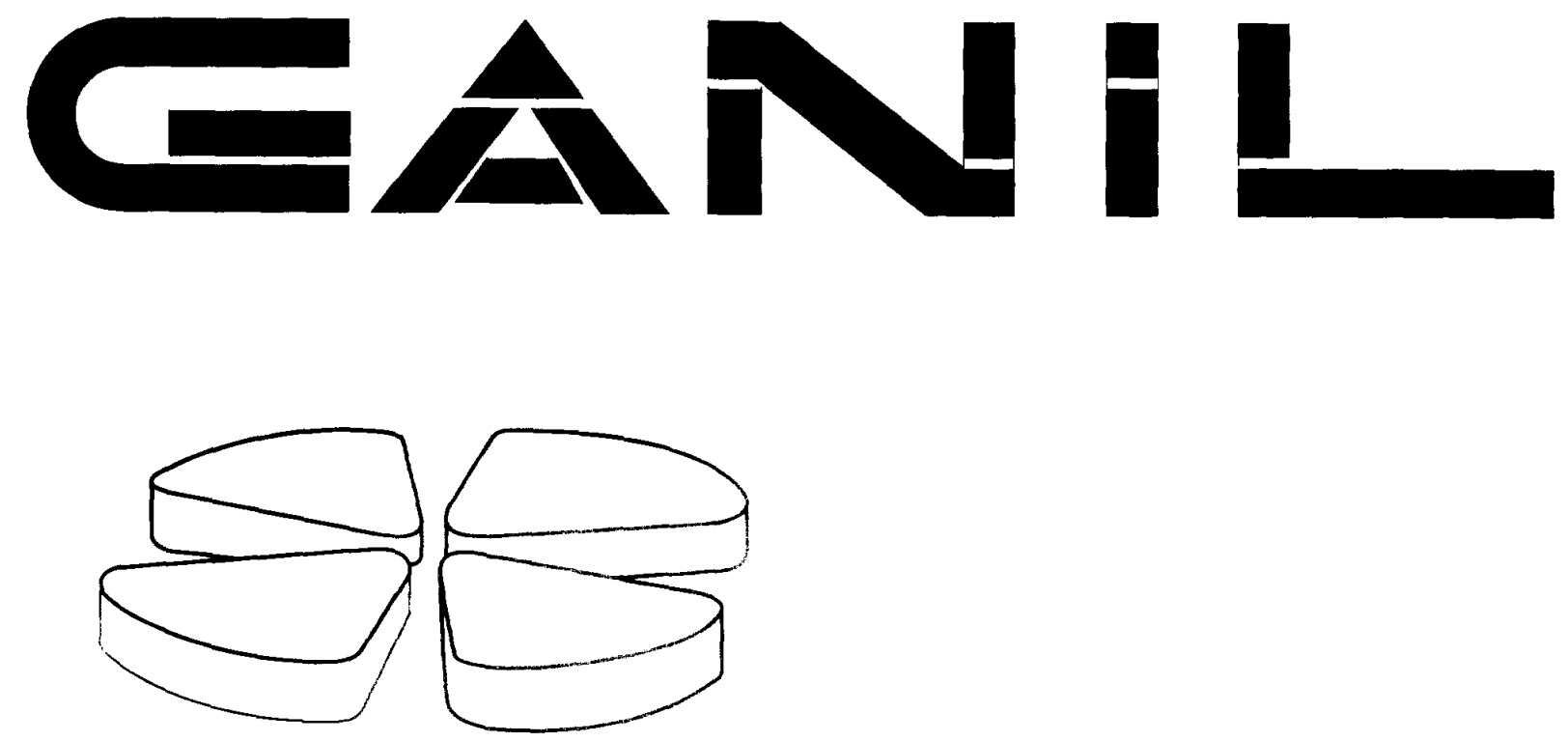

PROJECTILE COILOMB FXCITATION WITH FAST RADIOACTIVE BEAMS

R. Anne'), D. Bazin ', R. Bımbot", M.J.G. Borge ${ }^{8}$, J.M. Corre' , S. Dogny ${ }^{3 !}$, H. Eming ${ }^{4 !}$. D. Guillemaud-Mueller ${ }^{3}$, P.G. Hansen ${ }^{5 !}$, P. Hornshoj ${ }^{5 !}$. P. Jensen ${ }^{5)}$, B. Jonson ${ }^{2)}$, M. Lewitowicz ${ }^{11}$. A.C. Mueller ${ }^{3}$. R. Neugart 6), T. Nilsson ${ }^{2}$, G. Nyman ${ }^{2)}$, F. Pougheon ${ }^{3)}$, M.G. Saint-Laurent ${ }^{1}$, G. Schrieder ${ }^{7}$, O. Sorlin ${ }^{3}$, O. Tengblad ${ }^{8}$. K. Wilhelmsen-Rolander ${ }^{2}$ )

1) GANIL, BP 5027. F-14021 Caen

2) Fysiska Institutionen, Chalmers Tekniska Högskola, S-41296 Göteborg

3) Institut de Physique Nucléaire, CNRS-IN2P3, F-91406 Orsay

4) Gesellschaft für Schwerionenforschung (G.S.I.), D-64291 Darmstadt

5) Institut for Fysik og Astronomi, Aarhus Universitet, DK-8000 Aarhus $C$

6) Institut für Physik, Universität Mainz, D-55099 Mainz

7) Institut für Kernphysik, Technische Hochschule, D-64289 Darmstadt

8) CERN, Div. PPE, CH-1211 Genève 23

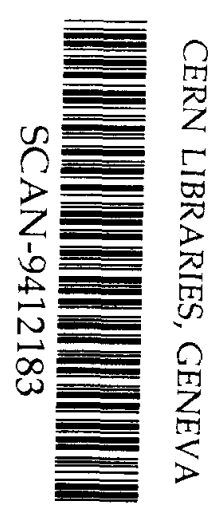

$\sin 3451$ 


\title{
PROJECTILE COULOMB EXCITATION WITH FAST RADIOACTIVE BEAMS
}

\author{
R. Anne ${ }^{1)}$, D. Bazin ${ }^{1)}$, R. Bimbot ${ }^{3)}$, M.J.G. Borge ${ }^{8)}$, J.M. Corre ${ }^{1)}$, S. Dogny ${ }^{3)}$, H. Emling ${ }^{4}$,
} D. Guillemaud-Mueller ${ }^{3)}$, P.G. Hansen ${ }^{5)}$, P. Hornsh $\varnothing^{5)}$, P. Jensen ${ }^{5)}$, B. Jonson ${ }^{2}$, M. Lewitowicz $^{1)}$, A.C. Mueller ${ }^{3)}$, R. Neugart 6), T. Nilsson ${ }^{2)}$, G. Nyman ${ }^{2)}$, F. Pougheon ${ }^{3)}$, M.G. Saint-Laurent ${ }^{1)}$, G. Schrieder ${ }^{7)}$, O. Sorlin ${ }^{3)}$, O. Tengblad ${ }^{8)}$, K. Wilhelmsen-Rolander ${ }^{2}$ )

\author{
1) GANIL, BP 5027, F-14021 Caen \\ 2) Fysiska Institutionen, Chalmers Tekniska Högskola, S-412 96 Göteborg \\ 3) Institut de Physique Nucléaire, CNRS-IN2P3, F-91406 Orsay \\ 4) Gesellschaft für Schwerionenforschung (G.S.I.), D-64291 Darmstadt \\ 5) Institut for Fysik og Astronomi, Aarhus Universitet, DK-8000 Aarhus $C$ \\ 6) Institut für Physik, Universität Mainz, D-55099 Mainz \\ 7) Institut für Kernphysik, Technische Hochschule, D-64289 Darmstadt \\ 8) CERN, Div. PPE, CH-1211 Genève 23
}

\section{$\underline{\text { Abstract }}$}

We report a search for $\gamma$ rays emanating from Coulomb excitation of fast (30-46 MeV/u) radioactive projectiles ${ }^{8} \mathrm{He},{ }^{11,12,14} \mathrm{Be}$ interacting with a lead target. These are clearly identified by their Doppler shift. The $320 \mathrm{keV} 1 / 2^{-} \longrightarrow 1 / 2^{+} \gamma$ transition from ${ }^{11} \mathrm{Be}$ was observed with a cross-section of $191 \pm 26 \mathrm{mb}$ which is noticeably less than expected from the known lifetime and in the perturbation limit of pure Coulomb excitation. In the other nuclei rather stringent upper limits of 0.01 to 0.2 Weisskopf units, are placed on the hypothetical transition to $1^{-}$states. 
An experiment on the Coulomb excitation of bound excited levels in nuclei having a neutron halo in their ground state has been performed. The work is a continuation of previous experiments at GANIL, in which nuclei with one $\left({ }^{11} \mathrm{Be}\right)$, two $\left({ }^{11} \mathrm{Li},{ }^{14} \mathrm{Be}\right)$ and four $\left({ }^{8} \mathrm{He}\right)$ halo neutrons have been investigated via their reaction cross-sections [1] and neutron angular distributions from the break-up of the neutron halo [2]. Just as in these experiments dealing with Coulomb excitation to the continuum, the present work is facilitated by the very high polarisability of the loosely bound halo, which gives rise to very large cross-sections for E1 excitation. Our test case is the nucleus ${ }^{11} \mathrm{Be}$, which so far is the only known halo system with a bound excited state (at $320 \mathrm{keV}$ ). The lifetime and hence the $\mathrm{B}(\mathrm{E} 1)$ value for this state are known from the Doppler-shift experiment of Millener et al.[3]. It was possible to populate this $1 / 2^{-}$state and to observe the transition back to the $1 / 2^{+}$ground state. Thus, for the first time, a Coulomb excitation experiment was achieved with a high energy $(45 \mathrm{MeV} / \mathrm{u})$ radioactive beam. We note for completeness two recent experiments [4,5] which by special techniques have obtained Coulomb excitation of slow radioactive projectiles.

The experiment was performed at GANIL's LISE3 [6] spectrometer with secondary beams obtained from the fragmentation of a primary $18 \mathrm{O}$ beam $(63 \mathrm{MeV} / \mathrm{u})$. Three independent criteria of LISE3 were used: (i) magnetic rigidity selection, (ii) energy loss selection with an achromatic wedge shaped degrader located at the dispersive focal plane and (iii) velocity selection by the Wien filter. Their combination permits the selection and transmission of secondary beams of ${ }^{11} \mathrm{Be}\left(45 \mathrm{MeV} / \mathrm{u}, 10^{5}\right.$ particles per second), ${ }^{12} \mathrm{Be}$ (46 MeV/u, $2.310^{4} \mathrm{pps}$ ), ${ }^{14} \mathrm{Be}(35 \mathrm{MeV} / \mathrm{u}, 250 \mathrm{pps})$ and ${ }^{8} \mathrm{He}(30 \mathrm{MeV} / \mathrm{u}, 500 \mathrm{pps})$ with a high isotopic purity (between 90 and $95 \%)$.

The experiment was based on a strategy similar to that of our recent experiments [2] studying the interplay between structure and reaction mechanism in the dissociation of ${ }^{11} \mathrm{Be}$ in collisions with light and heavy targets. The appearance of a fast ${ }^{10} \mathrm{Be}$ fragment with beam velocity was taken as indicating an impact parameter greater than $b_{\min }=R_{1}+R_{2}$, the sum of the core and target radii. As this work demonstrated, this does not exclude nuclear contributions to the reactions: the neutron halo extends far beyond $R_{1}$. In the present experiment, described in more detail in ref. [7], the good events were selected such that a gamma ray was detected in coincidence with the projectile, observed after the target. We assumed that at the high energies involved here the core-target collisions will lead to fragmentation of the projectile.

We used the experimental set-up shown in the figure 1 in which the radioactive beam impinges onto a lead target $\left(252 \mathrm{mg} / \mathrm{cm}^{2}\right)$. In order to accept high count rates, we used plastic scintillators (NE102) for charged particle identification and monitoring. The distance between the target and the plastic scintillators and the size of these detectors $\left(10 \times 10 \mathrm{~cm}^{2}\right)$ define roughly 
a cone with half-opening angle of $5^{\circ}$ : this should select the same minimum impact parameter $b_{\min }$ as in the previous experiment. Three large-volume germanium counters (70\% intrinsic efficiency for the $1332 \mathrm{keV}$ line of ${ }^{60} \mathrm{Co}$ ) detect in-flight $\gamma$ de-excitation. The Doppler shift of these $y$ rays depends on the angle of detection, and hence the distances from the target indicated in Fig.1 reflect a compromise between minimum Doppler broadening and maximum solid angle.

The $\gamma$ lines arising from projectile excitation are clearly distinguished from those from target excitation and from room background by their Doppler shift and the associated line broadening. Contours on two-dimensional particle identification spectra (energy loss vs. time of flight) eliminated contributions from the residual impurities contained in the secondary beam, and fragments produced in the target. A time window (100 ns) selected the gamma rays emitted in coincidence with a particle of the secondary beam resulting in strong suppression of background. The residual background of random coincidences showed some very weak $\gamma$ lines, identified as due to room background radioactivity, as well as some structures due to $\mathrm{Ge}\left(\mathrm{n}, \mathrm{n}^{\prime} \gamma\right)$ reactions.

We start with the results for the ${ }^{11} \mathrm{Be}$ shown in Fig. 2 . The peaks with shifted positions and large widths clearly must come from projectile Coulomb excitation: (i) the observed widths are consistent with calculations that take into account the Doppler broadening induced by the solid angle acceptance of the detectors, and by the momentum spread of the secondary radioactive beam, and (ii) as can be seen from Table 1, the positions of the centers of gravity observed in the experiment agree with the expected positions of the $320 \mathrm{keV}$ line. Those are calculated under the following assumptions: the mean energy of ${ }^{11} \mathrm{Be}$ at the middle of the target is derived from the magnetic rigidity of the second dipole of LISE and the energy loss in the Coulomb excitation target by means of the program STOPX [8]. We find a kinetic energy of $\mathrm{E}=43.0 \mathrm{MeV} / \mathrm{u}$ which gives a velocity $\beta=\mathrm{v} / \mathrm{c}=0.294$.

Relative to the number of incident ${ }^{11} \mathrm{Be}$ on NE102 plastic scintillators, the number of events in the $320 \mathrm{keV}$ peak detected in each germanium detector, corrected for geometrical and intrinsic efficiencies, yield to the differential cross-sections in the laboratory frame (Table 1). After kinematical corrections, a measured cross section of $191 \pm 26 \mathrm{mb}$ for the excitation of the $320 \mathrm{keV}$ level in the ${ }^{11} \mathrm{Be}$ was obtained, assuming an isotropic distribution in the centre-of-mass system consistent with the $1 / 2^{-} \longrightarrow 1 / 2^{+}$assignment (see below). The result can be compared with the adopted [3] lifetime of $166 \pm 15 \mathrm{fs}$, an average of three measurements, from which $\mathrm{B}(\mathrm{E} 1)=0.116 \pm 0.012 \mathrm{e}^{2} \mathrm{fm}^{2}$ is obtained. Under the assumption of pure first-order Coulomb excitation [9], this value leads to a predicted cross-section of $490 \pm 50 \mathrm{mb}$ for a $43 \mathrm{MeV} / \mathrm{u}{ }^{11} \mathrm{Be}$ beam incident on a lead target. 
In the case of ${ }^{12} \mathrm{Be}$ no gamma ray attributable to an excited $1^{-}$level was observed. In order to convert this result into quantitative limits, we have estimated the intensities that would lead to a detectable transition, first in a visual approach and then in a statistical analysis. Assuming Coulomb excitation to be dominant, we measure the intensities in Weisskopf units (W.u.) such as used in [3], that is with the statistical factor set to unity. If the statistical weight factor of 3, appropriate for the upgoing transition, were included, our limits would be correspondingly smaller. Fig. 3 summarizes such an analysis in the case of the detector " $90^{\circ}$ close" for the ${ }^{12} \mathrm{Be}$. Having set the reduced transition probability $\mathrm{B}(\mathrm{E} 1)$ to $0.2 \mathrm{~W}$.u. for the transition between an hypothetical level and the ground-state, we superimpose on the lower part of the figure the expected gaussians: the visual comparison excludes the existence of such a level.

The second step of our analysis is based on a $\chi^{2}$ procedure. Consider a segment of the spectrum, for exemple the energy range from 150 to $450 \mathrm{keV}$, shown in one of the insets. We fit it first with a simple exponential, for the case of a no-peak hypothesis, and obtain a certain value $\chi_{0}^{2}$. We then assume the presence of an additional peak, with the following characteristics: (i) centre of gravity $E_{\gamma}$, apparent energy of a transition $E_{\gamma_{0}}$, (ii) intensity defined by an imposed $\mathrm{B}(\mathrm{E} 1)$ value, and (iii) width calculated from the Doppler broadening. It is essential that the new adjustment also re-fits the background. The net result of a search for peaks with Ey and B(E1) varying respectively from $150 \mathrm{keV}$ to $1900 \mathrm{keV}$ and from 0.01 to $0.1 \mathrm{~W}$.u. is as follows: in the energy range $150-1000 \mathrm{keV}$ the upper limit is $0.01 \mathrm{~W}$.u. except near the energies $560-600 \mathrm{keV}$ and $830 \mathrm{keV}$, where transitions from inelastic neutron reactions on germanium or aluminium mask the signal at the level of about 0.03 W.u., both referred to the $90 \%$ confidence level. In the energy range $1000-2000 \mathrm{keV}$, the upper limit is $0.02 \mathrm{~W}$.u. Finally, a broadened line corresponding to $320 \mathrm{keV}$ centre-of-mass energy was seen in all three detectors at the expected energies and with an intensity close to $0.01 \mathrm{~W} . \mathrm{u} .$. We interpret this as arising from $\left({ }^{12} \mathrm{Be}\right.$, $\left.{ }^{11} \mathrm{Be}(320 \mathrm{keV})\right)$ stripping with a cross-section of $35 \pm 15 \mathrm{mb}$. For ${ }^{8} \mathrm{He}$ and ${ }^{14} \mathrm{Be}$ the statistics are lower, owing to weaker intensities of the radioactive beams. We find that, for the ${ }^{8} \mathrm{He}$, the upper limit varies from 0.05 to $0.1 \mathrm{~W} . \mathrm{u}$. in the energy range $150-2000 \mathrm{keV}$. For the ${ }^{14} \mathrm{Be}$, the upper limit varies from 0.1 to $0.2 \mathrm{~W} . \mathrm{u}$. in the energy range $150-1400 \mathrm{keV}$. In these limit calculations, we assume that full intensities of the radioactive beams were correctly detected.

We now turn to a discussion of the ${ }^{11} \mathrm{Be}$ result, interesting as well as puzzling: the cross-section for fast Coulomb excitation is only $40 \%$ of that deduced from the measured lifetime of the state on the basis of perturbation theory. As will be seen from the following discussion, this result remains difficult to interpret, and even if there is no indication of a possible error in the analysis, we feel that it should be regarded with some caution until it has been confirmed in a new experiment. We note incidentally that if the spin of the ${ }^{11} \mathrm{Be}$ ground 
state were to be $3 / 2$ instead of $1 / 2$, our result would agree with [3]. However, a large body of data is consistent with the spins and parities usually assumed for the bound states of this nucleus, and it is reassuring to realize that the assignments are unique. The essential links in the argument are: (i) the $(\mathrm{d}, \mathrm{p})$ reaction on ${ }^{10} \mathrm{Be}$ clearly [10] shows $l=0$ and $l=1$ for the 0 and 320 $\mathrm{keV}$ levels, respectively. This fixes the ground state as $1 / 2^{+}$. (ii) The gamma transition from the $320 \mathrm{keV}$ level is so fast (see above) that it can only be E1, which confirms the parity assignment from the $(d, p)$ reaction and fixes the possible spins of the upper level to either $1 / 2$ or $3 / 2$. (iii) An ingenious experiment by Deutsch et al. [11] on direct muon capture on ${ }^{11} \mathrm{~B}$ to the 320 level of ${ }^{11} \mathrm{Be}$ shows a time-dependence of the capture-to-muon-decay ratio that is only consistent with the nuclear spin sequence $3 / 2$ to $1 / 2$. The time dependence arises from two effects, the existence of a transition between the two hyperfine levels (with total angular momentum $F=1,2$ ) of muonic boron-11 and of an $F$ selection rule that leads to different capture-to-decay ratios for the two hyperfine levels if the initial and final spins are different. (iv) Finally, the same experiment [11] finds that the muon capture to the upper state is allowed and that the ground state is forbidden. This confirms the absolute parity assignments from (i).

The two states can, to a first approximation, be assigned as the $1 \mathrm{~s}_{1 / 2}$ and $0 \mathrm{p}_{1 / 2}$ shellmodel states, but complex-structure admixtures are essential for the inversion of the natural order and for the precise evaluation of transition matrix elements. For a discussion of this we refer to Millener et al. [3] who found that the transition probability is reduced to about one half by complex-structure contributions of which the dominant one is $d_{5 / 2} \rightarrow p_{3 / 2}$. See also the recent paper by Sagawa et al. [12].

The transition amplitudes for the $\mathrm{E} 1$ transitions in the halo nuclei approach unity for small impact parameters and deviations from perturbation theory are expected. For the dissociation cross section of the ${ }^{11} \mathrm{Be}$ ground state (41 MeV/u, gold target) this effect amounted to 4\% [2] after integration over the impact parameter. It therefore seemed possible that secondorder excitations to the continuum from the $320 \mathrm{keV}$ state could lead to a reduction of the calculated cross-section [13]. However, a coupled-channel calculation with the de Boer-Winther code and other estimates [14] show that this effect must be small.

A second possibility would be Coulomb-nuclear interference in the excitation of the 320 $\mathrm{keV}$ level of ${ }^{11} \mathrm{Be}$. This is possible because the halo wave function extends far beyond the core radius $R_{1}$. We have performed a calculation, which as its starting point assumes that the nuclear part of the interaction must be chosen so that it also accounts for the nuclear excitations of the ground state of ${ }^{11} \mathrm{Be}$ to the continuum. These are appreciable. The calculations in the sudden approximation given in the last paper of ref. [2] found a nuclear contribution of $0.6 \mathrm{~b}$ to the total calculated dissociation cross-section of $3.0 \mathrm{~b}$ at $41 \mathrm{MeV} / \mathrm{u}$ on a gold target. The same calculation gives good agreement with experiment for the light targets, for which the nuclear 
contributions are dominant. We extend this model to include E1 excitations to the bound excited level. Since there is no equivalent to the zero-range approximation for a $\mathrm{p}$ state, we have used a finite square well with depth adjusted separately to reproduce the experimental neutron separation energies of the two states in question. The dissociation cross-sections calculated with the s-state wave function are in good agreement with those obtained previously and confirm the validity of the "finite-size correction" used in this paper. For the gamma transition amplitude we include the appropriate Clebsch-Gordan coefficient to take the spin into account. The calculation in the sudden approximation gives an excitation cross-section of $387 \mathrm{mb}$. The same calculation but with a series expansion of the interaction (dipole approximation) gives $8 \%$ more and the nuclear term, the "wound", carries with it a reduction of $8 \%$. The total effect of $-16 \%$ is still well below the value $-60 \%$ suggested by the experiment.

Other estimates of this effect were obtained in a coupled-channel calculation done by $\mathrm{N}$. Alamanos [15] with the ECIS code [16]. The nuclear transition potential is chosen according to Satchler's prescriptions [17], for which the essential parameter is the $\Delta R_{n p}$ value, the difference between the radii of the neutron and proton distributions. The Coulomb cross-section is in this case $518 \mathrm{mb}$ and the nuclear interference is here constructive, of the order of $2-7 \%$ (if $\Delta R_{n p}$ varies from 1 to $4 \mathrm{fm}$ ).

The search for $1^{-}$excited levels gave upper limits of the order of 0.01 to $0.2 \mathrm{~W} . \mathrm{u}$. for ${ }^{8} \mathrm{He}$ and ${ }^{12,14} \mathrm{Be}$ nuclei. Since the expected E1 transition strength is approaching one W.u., this strongly suggest there are no low-lying $1^{-}$state in the three even-even projectiles.

We finally, à propos the halo, draw attention to an interesting inequality discovered by Bertlmann and Martin [18]. It links the mean-square radius $\left\langle r^{2}>_{0}\right.$ of an $N$-body system of particles with mass $m$ to the excitation energy of the lowest $l=1$ state:

$$
\mathrm{E}_{1}-\mathrm{E}_{0} \leq \frac{3 \mathrm{~h}^{2}}{2 m<r^{2}>0}
$$

and is obtained from an argument involving essentially the Thomas-Reiche-Kuhn sum rule. It applies both for particles bound to a heavy core and to a system of identical particles interacting through attractive forces. For most simple, quantum mechanical system the bound is close to an equality, but nuclear physics shows that states with a complex structure can give much smaller values. The first state with opposite parity to the ground state in ${ }^{224} \mathrm{Ra}$ is found at an energy of $16 \%$ of that given by the Bertlmann-Martin bound, and for ${ }^{11} \mathrm{Be}$ the ratio is as low as $4 \%$.

The approach described here should be immediately applicable to the many fast radioactive beams that now exist around the world. With ${ }^{11} \mathrm{Be}$ ions of intermediate energy (43 $\mathrm{MeV} / \mathrm{u})$ and relatively low intensity $\left(10^{5} \mathrm{pps}\right)$, it has been possible to observe the Coulomb 
excitation of this halo nucleus with a, by modern standards, very modest detection system. Meaningful limits have been set for the nuclei ${ }^{12,14} \mathrm{Be}$ and ${ }^{8} \mathrm{He}$.

\section{Acknowledgements}

We would like to thank F. Geoffroy, R. Hue and L. Petizon for their technical assistance during the experiment, N. Alamanos, G. Baur aud C. Bertulani for discussions and R. Lombard for drawing our attention to the Bertlmann-Martin bond.

This work was partly supported by la Région Basse Normandie. One of us, G. Schrieder, would like to thank for the support by the German Federal Minister for Research and Technology (BMFT) under contract 06DA641. 


\section{References}

[1] M.G. Saint-Laurent et al., Z Phys. A332 (1989) 457; A.C.C. Villari, Phys. Lett. B268 (1991) 345.

[2] R. Anne et al., Phys. Lett. B250 (1990) 19; K. Riisager et al., Nucl. Phys. A540 (1992) 365;

R. Anne et al., Phys. Lett. B304 (1993) 55-59; R. Anne et al., Nucl. Phys. A575 (1994) 125-154.

[3] D.J. Millener et al, Phys. Rev C 28 (1983) 497.

[4] J.A. Brown et al,. Phys. Rev. Lett. 66 (1991) 2452-2455.

[5] M. Oshima et al, NIM A312 (1992) 425-430.

[6] A.C. Mueller and R.Anne, Nucl. Inst. Meth. B56 (1991) 559.

[7] J.M. Corre, thèse de l'Université de Caen (1994) GANIL T 9403.

[8] T. Awes, Oak Ridge Natinal Laboratory (1983).

[9] A. Winther et K. Alder, Nucl.Phys. A319 (1979) 518-532.

[10] D.R. Goosman and R.W. Kavanagh, Phys. Rev. C1 (1970) 1939; D.L. Auton, Nucl. Phys. A157 (1970) 305; B. Zwieglinski et al., Nucl. Phys. A315 (1979) 124.

[11] J.P. Deutsch et al., Phys. Lett. B28 (1968) 178.

[12] H. Sagawa et al., Phys. Lett. B309 (1993) 1.

[13] G. Baur and S. Typel, private communication, (1994).

[14] H.J. Wollersheim, private communication, (1993).

[15] N. Alamanos, private communication, (1994).

[16] J. Raynal, Phys. Rev C 23 (1981) 2571.

[17] G.R. Satchler, Nucl.Phys. A472 (1987) 215.

[18] R.A. Bertlmann and A. Martin, Nucl.Phys. B182 (1981) 35. 


\section{Table1}

Expected and experimental energies for the Doppler-shifted $320 \mathrm{keV}$ gamma-line, and differential cross-sections in the laboratory frame

\begin{tabular}{cccc}
\hline Detector & $\mathrm{E} \gamma=\frac{\mathrm{E} \gamma_{0} \sqrt{1-\beta^{2}}}{1-\beta \cos \theta}(\mathrm{keV})$ & $\mathrm{E} \gamma(\mathrm{exp})(\mathrm{keV})$ & $\frac{\mathrm{d} \sigma}{\mathrm{d} \Omega_{\gamma}} \mathrm{lab}(\mathrm{mb} / \mathrm{str})$ \\
\hline $55^{\circ}$ & 367,9 & $369,3 \pm 0,6$ & $16,1 \pm 3,5$ \\
$90^{\circ} \mathrm{close}$ & 305,9 & $306,9 \pm 0,5$ & $15,8 \pm 3,6$ \\
$90^{\circ} \mathrm{far}$ & 305,9 & $308,7 \pm 0,2$ & $11,6 \pm 2,9$ \\
\hline
\end{tabular}




\section{Figure captions.}

Fig. 1 - Experimental arrangement.

Fig. 2 - Gamma spectra in the relevant energy-range for the $320 \mathrm{keV}$ transition in ${ }^{11} \mathrm{Be}$. The vertical line shows the expected position of the $E \gamma_{0}=320 \mathrm{keV}$ peaks when de-excitating nuclei are at rest. In the present case of fast moving projectiles, a large Doppler shift is present. At $90^{\circ}$ we observe the influence of second order contribution (see Table 1). The widths are dependent on the target-detector distances and exceed the detector resolution ( $3 \mathrm{keV}$ at $1332 \mathrm{keV}$ ). The strong continuous background arises from $\gamma$ rays from reactions in material along the beam path such as the exit window of the spectrometer and the plastic scintillators themselves.

Fig. 3 - Search for peaks in the spectrum of the " $90^{\circ}$ close" germanium detector, for the case of the ${ }^{12} \mathrm{Be}$ radioactive beam. In the lower part of this picture, superimposed to the experimental spectrum, are shown the expected line shapes for hypothetical levels of different energies $\left(\mathrm{E} \gamma_{0}=320 \mathrm{keV}, 500 \mathrm{keV}, \ldots\right)$, all with the same reduced transition probability equal to 0.2 W.u. Note how the line shape clearly distinguishes the gamma rays due to projectile excitation from those in the background. The inset summarizes, in terms of contour plots, the $\chi^{2}$ procedure described in the text. 


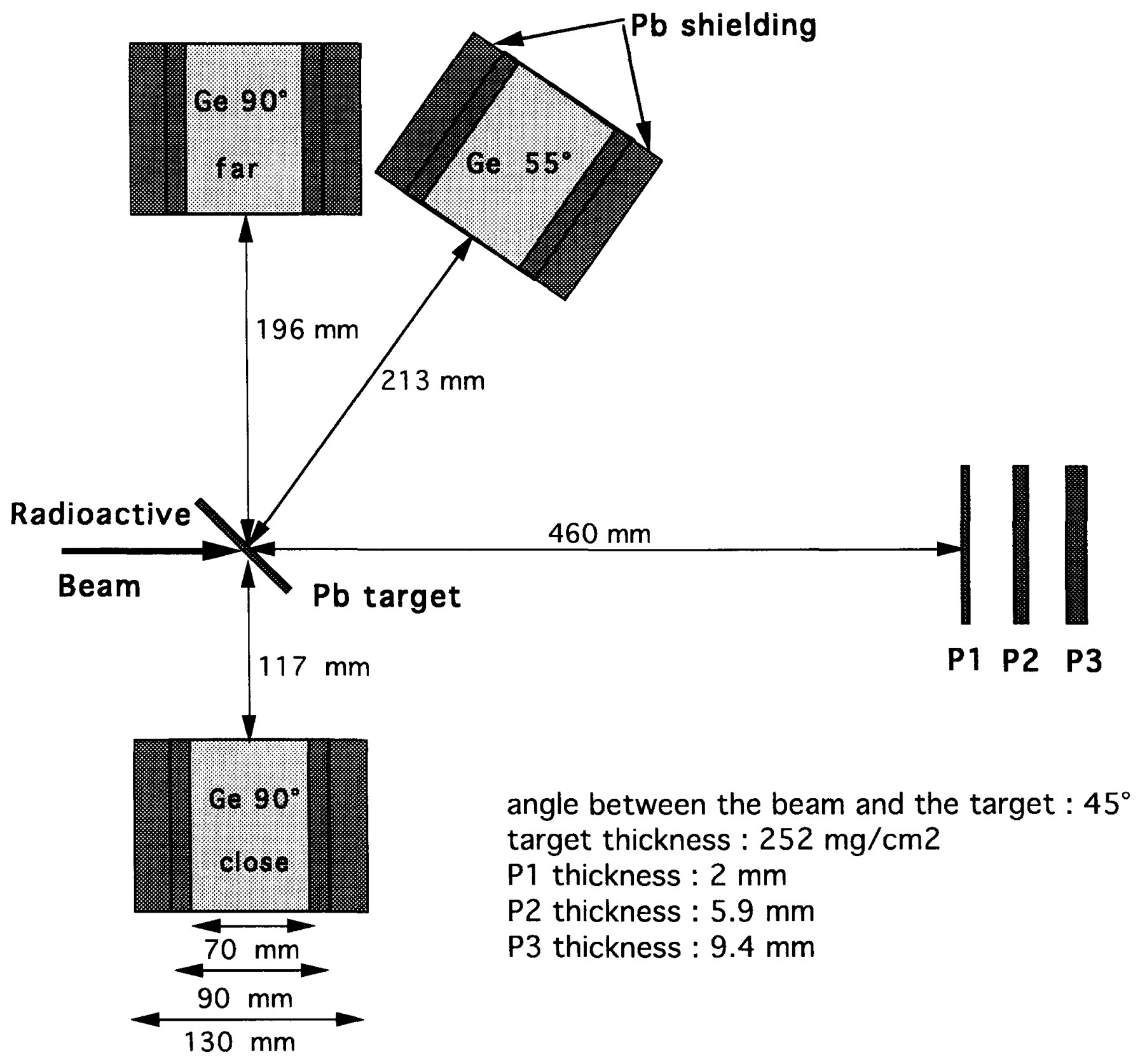

Fig. 1 


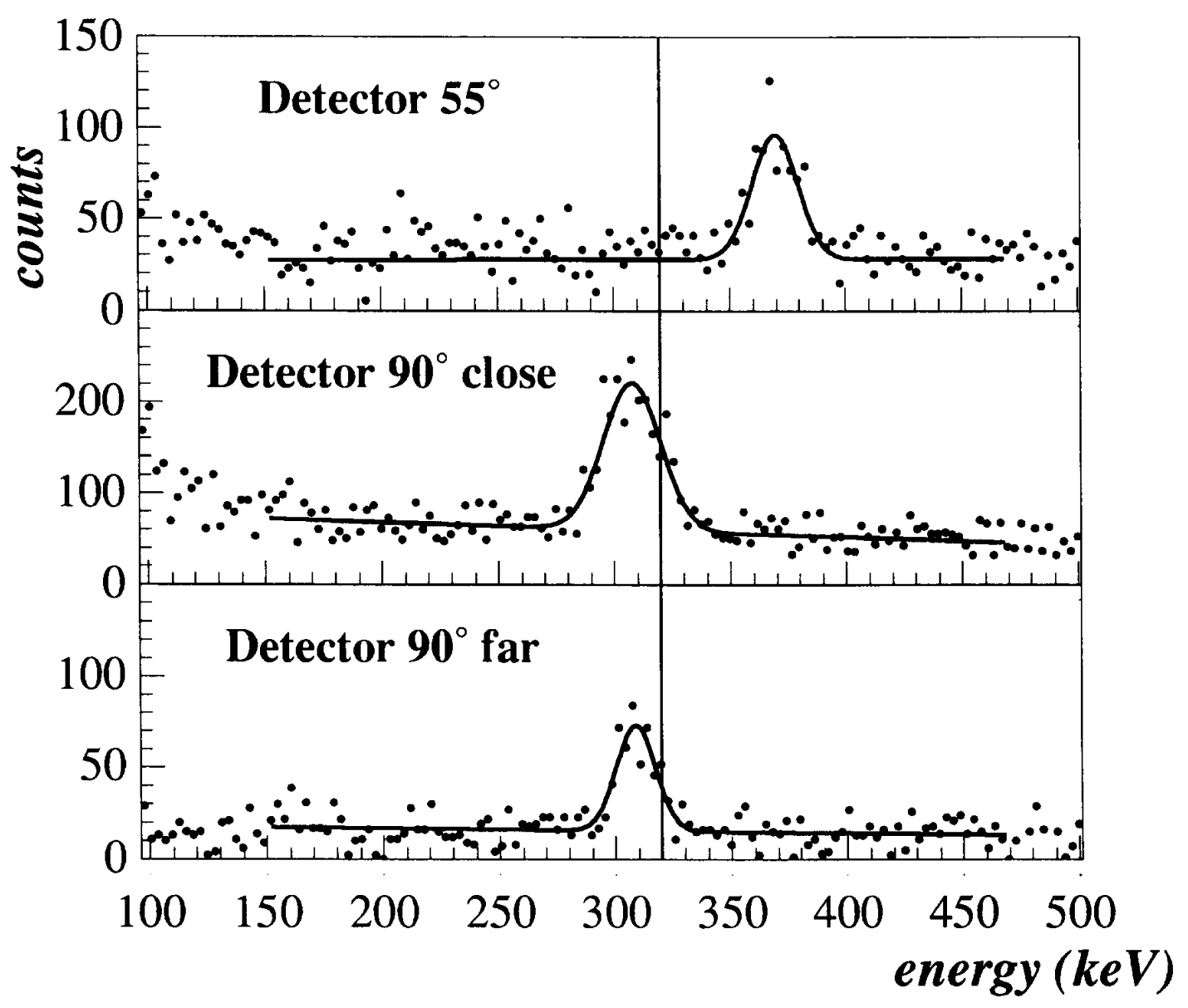

Fig. 2 


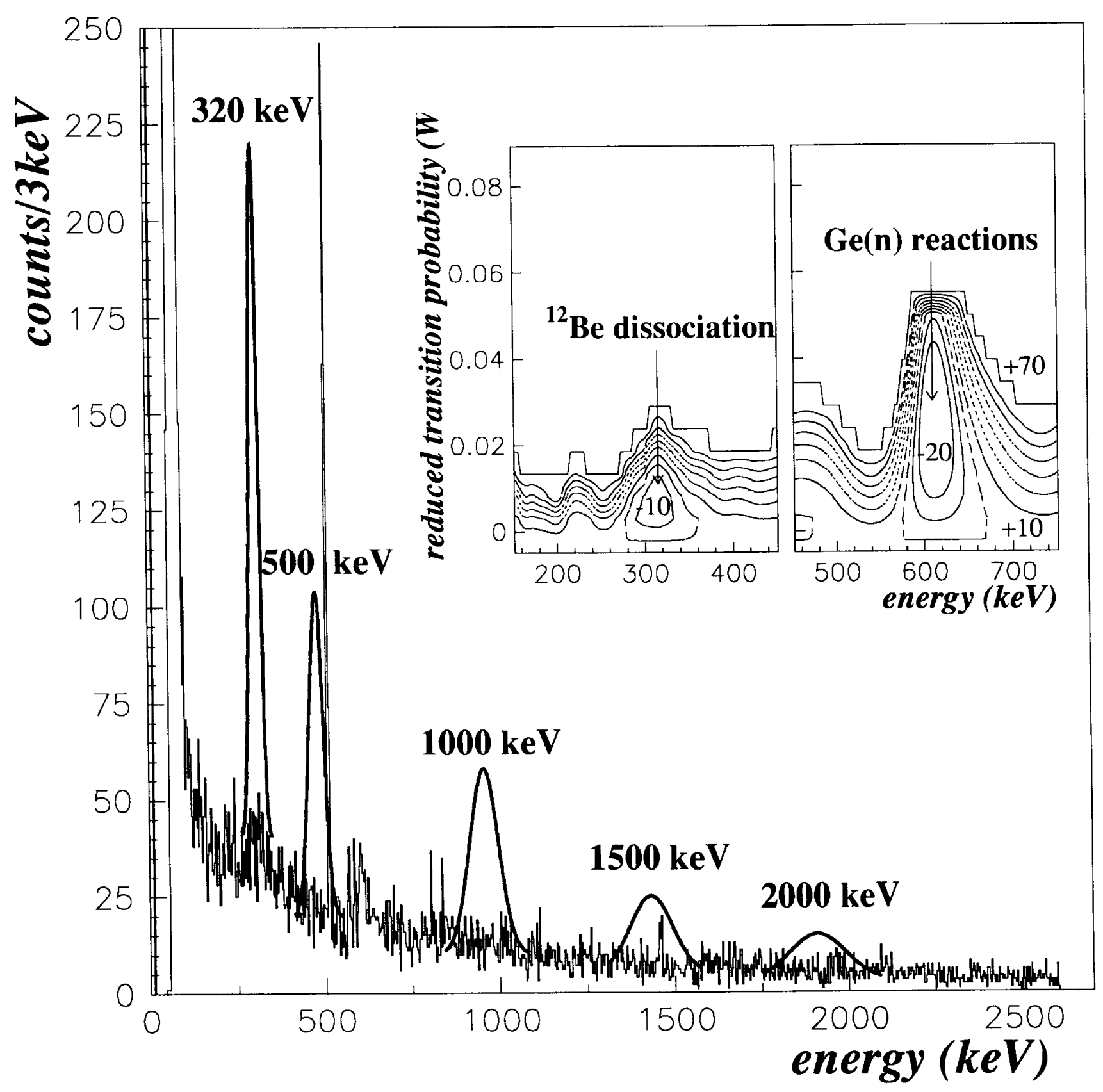

Fig. 3 
